ARTÍCULOS ORIGINALES Rev Chil Salud Pública 2014; Vol 18 (2): 140-148

\section{CONOCIMIENTO, ACTITUDES Y PRÁCTICAS EN SALUD ORAL EN ADOLESCENTES DE 12 Y 15 AÑOS DE LA LOCALIDAD DE FRESIA, AÑO 2013}

\author{
Oral health KNOWLeDGe, ATtITUDES AND habits OF \\ ADOLESCENTS FROM FrESIA AGED 12 AND 15, IN 2013
}

\section{RESUMEN}

Introducción. Los adolescentes muestran escasos conocimientos sobre las enfermedades bucales más comunes. En Chile, en la Encuesta Nacional de Calidad de Vida realizada el año 2006, un 37\% de los jóvenes mayores de 15 años, dijo sentir que su salud bucal afecta su calidad de vida. Para planificar y llevar a cabo actividades de promoción de la salud oral y modificar las conductas de riesgo, es importante saber cuál es el conocimiento existente, actitudes y prácticas de este grupo.

Objetivo. Determinar el nivel de conocimiento, actitudes y prácticas en salud oral, de adolescentes de 12 y 15 años, de establecimientos educacionales de Fresia, año 2013.

Material y Métodos. Se realizó un estudio observacional descriptivo, por medio de una encuesta a los adolescentes de 12 y 15 años de los establecimientos educacionales de Fresia, previa autorización de los apoderados. La encuesta constó de 25 preguntas de verdadero/falso y de selección múltiple.

Resultados. Se encuestaron 170 adolescentes, de ellos un 81,8\% cree que cepillarse los dientes ayuda a prevenir la caries, un 42,4\% declaró que el cepillado dental no previene las enfermedades de las encías, un 87,4\% piensa que su propio esfuerzo es el factor más importante para una buena salud oral. El $54 \%$ de los jóvenes dijo cepillarse en la mañana y en la noche.

Conclusión. Los adolescentes de 12 y 15 años de la localidad de Fresia tienen un nivel de conocimiento de salud oral aceptable, sin embargo, se debe reforzar la educación de las enfermedades que afectan a las encias. En relación a las actitudes y prácticas los resultados son favorables.

Palabras clave: adolescentes, conocimiento, actitudes, prácticas, salud oral.

\section{ABSTRACT}

Introduction: Adolescents show little knowledge of common oral diseases. In Chile, the National Survey on Quality of Life 2006, thirty-seven percent of 
teens aged 15 said they felt that their oral health affects their quality of life. To plan and carry out activities to promote oral health and change risk behaviors, it is important to know the existing knowledge, attitudes and practices of this group.

Objective. To determine the level of knowledge, attitudes and habits of oral bealth in adolescents aged 12 and 15, from educational establishments in Fresia, Chile, in 2013.

Materials and Methods. We conducted a descriptive study through a survey of adolescents (12 and 15) with prior approval from parents or carers. The survey consisted of 25 true/false and multiple-choice questions.

Results. 170 adolescents were surveyed. $81.8 \%$ believed that brushing belps prevent cavities, $42.4 \%$ stated that tooth brushing does not prevent gum disease, $87.4 \%$ believe that their own effort is the most important factor for good oral health. 54\% of young people confirmed brushing their teeth both morning and night.

Conclusion. Adolescents from the city of Fresia aged 12 and 15 years have an acceptable level of oral health knowledge. However, education of diseases affecting the gums should be strengthened. Regarding attitudes and practices, the results are favorable.

Key words: adolescents, knowledge, attitudes, practices, oral health.

\section{INTRODUCCIÓN}

Las enfermedades orales califican como importantes problemas de salud pública, debido a su alta prevalencia e incidencia en todas las regiones del mundo. La OMS propone a los jóvenes de 12 y 15 años, para la vigilancia de la patología bucal. ${ }^{1}$

En Chile, en la Encuesta Nacional de Calidad de Vida realizada el año 2006, un 37\% de los jóvenes mayores de 15 años, dijo sentir que su salud bucal afecta su calidad de vida siempre o casi siempre. El conocimiento es considerado la base para poder determinar actitudes, prácticas y comportamiento futuro que la persona adquiera en su diario vi$\mathrm{vir}^{2}{ }^{2}$ teniendo las educaciones en salud oral un impacto positivo en el conocimiento de la misma. ${ }^{3}$

Un estudio determinó que los adolescentes muestran escasos conocimientos sobre las enfermedades bucales más comunes y sus métodos de prevención. ${ }^{4}$ Se dice que los niños con insuficiente conocimiento de la salud oral son dos veces más propensos a tener caries. ${ }^{5} \mathrm{Se}$ observaron correlaciones significativas entre las condiciones de higiene oral, las percepciones y actitudes hacia la salud oral. ${ }^{6}$ En la localidad de Fresia, no se han realizado estudios similares en adolescentes.
La adolescencia es un período crítico de las prácticas de salud y suelen persistir durante la edad adulta.?

Para planificar y llevar a cabo actividades de promoción de la salud oral y modificar las conductas de riesgo, es importante saber cuál es el conocimiento, actitudes y prácticas existentes en este grupo. ${ }^{8}$

El objetivo de este estudio es determinar el nivel de conocimiento, actitudes y prácticas en salud oral, de adolescentes de 12 y 15 años, de establecimientos educacionales de la localidad de Fresia.

\section{METODOLOGÍA}

Se realizó un estudio descriptivo de tipo censal, basado en una encuesta aplicada a adolescentes de 12 y 15 años de los establecimientos educacionales de la ciudad de Fresia, entre los meses de septiembre y octubre del año 2013. Este estudio contó con la aprobación de la Ilustre Municipalidad de Fresia.

La encuesta realizada fue adaptada de los Cuestionarios para Niños, diseñada por la OMS. ${ }^{8}$ Para ello, un investigador (PB) realizó la traducción de la encuesta al idioma español. Luego, se presentó a docentes y estudiantes de los distintos establecimientos educacionales 
para obtener, mediante preguntas abiertas, la opinión de su fachada e interpretación de las preguntas. Posteriormente, tres investigadores (CB, PB y AP), mediante consenso, realizaron las modificaciones y adaptaciones lingüísticas de la encuesta original. Finalmente, se aplicó la encuesta a un grupo piloto que representaba el $10 \%$ de la muestra total, 24 adolescentes de 12 y 15 años, escogidos al azar, los cuales no participaron en el estudio final. Se determinó el número de dominios e ítems de la encuesta, obteniendo finalmente un instrumento con cuatro dominios: 1) Conocimiento sobre salud oral, 2) Actitudes relacionadas con salud oral, 3) Prácticas relacionadas con salud oral y 4) Características sociodemográficas. La encuesta tuvo un total de 25 ítems, 11 de verdadero y falso y 14 de alternativas, como se muestra en la Tabla 1 (Anexo 1).

Los criterios de inclusión considerados fueron todos los adolescentes de 12 y 15 años, de los establecimientos educacionales Escuela Básica Fresia, Colegio Purísimo Corazón de María y Liceo Carlos Ibáñez del Campo. Los criterios de exclusión utilizados fueron aquellos que no presentaron el consentimiento informado firmado por su apoderado y personas inhabilitadas física o mentalmente para responder la encuesta.

Se solicitó a la Dirección de cada establecimiento, las listas de los cursos sexto, séptimo y octavo básico y primero y segundo medio, para seleccionar a los adolescentes de 12 y 15 años. Se realizaron dos visitas, en el mes de septiembre y octubre del año 2013, en las salas de clases de cada curso con adolescentes seleccionados. En la primera visita se les explicó en qué consistía el estudio, para luego proceder a entregarles el consentimiento informado, para ser firmado por sus apoderados, por ser ellos menores de edad. En la segunda visita se retiraron los consentimientos informados y a aquellos adolescentes que aceptaron participar se les solicitó que contestaran la encuesta, la cual se aplicó de forma presencial, anónima y voluntaria, en formato papel, no contemplando fines de lucro.

Para disminuir el efecto Hawthorne y así un posible sesgo, se les pidió encarecidamente que contestaran con honestidad, recordándoles que toda la información se manejaría en confidencialidad, mientras eran supervisados por los investigadores.

Los datos obtenidos fueron ingresados a una planilla Google Docs. por los investigadores. Se realizó estadística descriptiva, utilizando el Paquete Estadístico SPSS 20 (IMB, Chicago, EE.UU.), para las variables:

1. Conocimientos en salud oral: Caries. Influencia del cepillado dental. Visitas al odontólogo. Hilo dental y flúor en caries, gingivitis. Importancia de los dientes naturales.

2. Actitudes relacionadas con salud oral: Cuidado e importancia de la salud bucal.

3. Prácticas realizadas en salud oral: Uso de cepillos. Elementos utilizados en el cepillado. Tipo de pasta de dientes. Tiempo y duración del cepillado dental. En qué momento del día se realiza el cepillado. Frecuencia de recambio del cepillo de dientes. Técnica de cepillado.

4. Características sociodemográficas
a. Sexo: femenino/masculino
b. Edad: 12 o 15 años
c. Residencia:

- Urbana: adolescentes que viven en la ciudad de Fresia

- Rural: adolescentes que viven en las localidades aledañas a la ciudad de Fresia, como Parga, Tegualda, La Isla, entre otras.

\section{RESULTADOS}

Las características sociodemográficas de la muestra en estudio se encuentran resumidas en la Tabla 2.

De los tres establecimientos educacionales contactados para participar en el estudio, se obtuvo un total de 170 adolescentes encuestados, la mayoría mujeres $(52,4 \%)$ de 12 años $(73,3 \%)$.

De los estudiantes, un $95,9 \%$ dijo que las bacterias causan caries en los dientes; un 
Tabla 2. Características sociodemográficas de los adolescentes en estudio $(n=170)$

\begin{tabular}{|c|c|c|c|c|c|c|}
\hline & & Sexo & & Residencia & & Total (\%) \\
\hline \multirow{3}{*}{ Edad } & & Masculino (\%) & Femenino $(\%)$ & Urbano (\%) & Rural (\%) & \\
\hline & 12 & $42(56,0)$ & $33(44,0)$ & $55(73,3)$ & $20(26,7)$ & $75(100)$ \\
\hline & 15 & $39(41,1)$ & $56(58,9)$ & $50(52,6)$ & $45(47,4)$ & $95(100)$ \\
\hline Total & & $81(47,6)$ & $89(52,4)$ & $105(61,8)$ & $65(38,2)$ & $170(100)$ \\
\hline
\end{tabular}

Tabla 3. Conocimiento de estudiantes de 12 y 15 años en salud oral pertenecientes a establecimientos educacionales de la ciudad de Fresia $(n=170)$

\begin{tabular}{lrrr}
\hline \multicolumn{1}{c}{ Variable } & Verdadero (\%) & Falso (\%) & No sé (\%) \\
\hline 1. Las bacterias (gérmenes) en los dientes causan caries & $63(95,9)$ & $1(0,6)$ & $6(3,5)$ \\
2. Los dientes naturales son mejores que los dientes postizos (falsos) & $140(82,4)$ & $19(11,2)$ & $11(6,5)$ \\
3. Cepillarse los dientes puede prevenir las caries & $139(81,8)$ & $28(16,5)$ & $3(1,8)$ \\
4. Comer y beber alimentos dulces no provoca caries & $19(11,2)$ & $145(85,3)$ & $6(3,5)$ \\
5. Debes visitar al dentista al menos una vez al año, incluso si no tienes problemas & $140(82,4)$ & $15(8,8)$ & $15(8,8)$ \\
o dolor en los dientes & & & $38(22,4)$ \\
6. Usar hilo dental puede prevenir las caries & $85(50)$ & $47(27,6)$ & $69(40,6)$ \\
7. El uso de flúor previene las caries & $70(41,2)$ & $31(18,2)$ & $17(10,0)$ \\
8. El sangrado en el cepillado puede ser un signo de enfermedad en las encías & $129(75,9)$ & $24(14,1)$ \\
9. La enfermedad de las encías se puede producir por falta de higiene dental & $118(69,4)$ & $8(4,7)$ & $44(25,9)$ \\
10. Cepillarse los dientes no previene problemas de las encías & $66(38,8)$ & $72(42,4)$ & $32(18,8)$ \\
11. Mantener los dientes naturales no es tan importante & $8(4,7)$ & $152(89,4)$ & $10(5,9)$ \\
\hline
\end{tabular}

$85,3 \%$ asoció también los alimentos dulces como factor causal. Un $82,4 \%$ piensa que se debe visitar al dentista al menos una vez al año, incluso si no se tienen problemas o dolor en los dientes. En cuanto a la prevención de la caries, un $81,8 \%$ cree que es de utilidad el cepillado dental, un $50 \%$ dice lo mismo en relación al uso de hilo dental y un 41,2\% con respecto al flúor.

En cuanto a la enfermedad de las encías (gingivitis), un 69,4\% la asoció con gérmenes de los dientes, un 75,9\% cree que el sangrado en el cepillado puede ser un signo de esta enfermedad y un $42,4 \%$ considera que el cepillado dental sirve para su prevención. Un $89,4 \%$ de los adolescentes cree que mantener los dientes naturales es importante y un $82,4 \%$ dice que estos son mejores que los dientes protésicos (Tabla 3 ).

En cuanto a las actitudes de los adolescentes relacionadas con la salud oral, un 40,6\% declaró tener un buen cuidado de sus dien- tes y un $34,7 \%$ cree tener un conocimiento regular sobre enfermedades de las encías. Un $72,9 \%$ de los jóvenes considera muy importante limpiar sus dientes, declarando el $68,2 \%$ que es muy importante tener condiciones bucales saludables. Un $84,7 \%$ cree que sus propios esfuerzos son el factor más importante para el futuro de su salud oral. En la Tabla 4 se muestran las actitudes que tienen los adolescentes frente a su salud oral distribuidos por edad.

En relación a las prácticas en salud oral de los adolescentes, el 100\% relató usar cepillo de dientes y que lo complementa con agua y pasta de dientes. El 50,6\% sabe que su pasta dental contiene flúor. En cuanto al cepillado dental, el 67,1\% de los jóvenes declaró cepillarse los dientes por más de tres minutos, realizando movimientos de arriba a abajo (60\%) y el $54,1 \%$ de los casos, lo hace en la mañana y en la noche. Un $64,1 \%$ de estos adolescentes, declara cambiar su cepillo de dientes en 
Tabla 4. Actitudes de adolescentes de 12 y 15 años en salud oral pertenecientes a establecimientos educacionales de la ciudad de Fresia $(n=170)$

\begin{tabular}{|c|c|c|c|c|}
\hline \multirow{2}{*}{ Variable } & & \multicolumn{2}{|c|}{ Edad (\%) } & \multirow{2}{*}{$\begin{array}{c}\text { Total }(\%) \\
(\mathrm{n}=170)\end{array}$} \\
\hline & & $12(n=75)$ & $15(\mathrm{n}=95)$ & \\
\hline \multirow{4}{*}{$\begin{array}{l}\text { 1. Qué tan bien crees que tomas el } \\
\text { cuidado de tus dientes }\end{array}$} & Muy bien & $15(20,0)$ & $16(16,8)$ & $31(8,2)$ \\
\hline & Bien & $24(32,0)$ & $45(47,4)$ & $69(40,6)$ \\
\hline & Regular & $34(45,3)$ & $33(34,7)$ & $67(39,4)$ \\
\hline & Mal & $2(2,7)$ & $1(1,1)$ & $3(1,8)$ \\
\hline \multirow{4}{*}{$\begin{array}{l}\text { 2. Mi conocimiento sobre } \\
\text { enfermedades de las encías es }\end{array}$} & Muy bueno & $22(29,3)$ & $16(16,8)$ & $38(22,4)$ \\
\hline & Bueno & $24(32,0)$ & $3334,7)$ & $57(33,5)$ \\
\hline & Regular & $21(28,0)$ & $38(40,0$ & $59(34,7)$ \\
\hline & No tengo conocimiento & $8(10,7)$ & $8(8,4)$ & $16(9,4)$ \\
\hline \multirow{3}{*}{$\begin{array}{l}\text { 3. Cuán importante es para ti limpiar } \\
\text { tus dientes }\end{array}$} & Muy importante & $55(73,3)$ & $69(72,6)$ & $124(72,9)$ \\
\hline & Importante & $20(26,7)$ & $25(26,3)$ & $45(26,5)$ \\
\hline & Poco o nada importante & $0(0,0)$ & $1(1,1)$ & $1(0,6)$ \\
\hline \multirow{3}{*}{$\begin{array}{l}\text { 4. Cuán importante es para ti tener } \\
\text { condiciones bucales saludables }\end{array}$} & Muy importante & $48(64,0)$ & $68(71,6)$ & $116(68,2)$ \\
\hline & Importante & $27(36,0)$ & $27(28,4)$ & $54(31,8)$ \\
\hline & Poco o nada importante & $0(0,0)$ & $0(0,0)$ & $0(0,0)$ \\
\hline \multirow{2}{*}{$\begin{array}{l}\text { 5. Cuál es el factor más importante } \\
\text { para el futuro de tu salud oral }\end{array}$} & Mis propios esfuerzos & $61(81,3)$ & $83(87,4)$ & $144(84,7)$ \\
\hline & El dentista & $14(18,7)$ & $12(12,6)$ & $26(15,3)$ \\
\hline
\end{tabular}

Tabla 5. Prácticas de adolescentes de 12 y 15 años en salud oral pertenecientes a establecimientos educacionales de la ciudad de Fresia $(n=170)$

\begin{tabular}{|c|c|c|c|c|}
\hline & \multirow{2}{*}{ Variable } & \multicolumn{2}{|c|}{ Edad (\%) } & \multirow{2}{*}{$\begin{array}{c}\text { Total }(\%) \\
(\mathrm{n}=170)\end{array}$} \\
\hline & & $12(\mathrm{n}=75)$ & $15(\mathrm{n}=95)$ & \\
\hline \multirow{2}{*}{ 1. Uso del cepillo de dientes } & Sí & $75(100)$ & $95(100)$ & $170(100)$ \\
\hline & No & $0(0,0)$ & $0(0,0)$ & $0(0,0)$ \\
\hline \multirow{3}{*}{$\begin{array}{l}\text { 2. Elementos utilizados para } \\
\text { cepillarte }\end{array}$} & Agua & $0(0,0)$ & $0(0,0)$ & $0(0,0)$ \\
\hline & Pasta de dientes + agua & $75(100)$ & $95(100)$ & $170(100)$ \\
\hline & Nada & $0(0,0)$ & $0(0,0)$ & $0(0,0)$ \\
\hline \multirow[t]{3}{*}{ 3. Tipo de pasta de dientes } & Fluorada & $31(41,3)$ & $55(57,9)$ & $86(50,6)$ \\
\hline & No fluorada & $4(5,3)$ & $3(3,2)$ & $7(4,1)$ \\
\hline & No lo sé & $40(53,3)$ & $37(38,9)$ & $77(45,3)$ \\
\hline \multirow{2}{*}{$\begin{array}{l}\text { 4. Tiempo de duración del } \\
\text { cepillado de dientes }\end{array}$} & Menos de 3 minutos & $29(38,7)$ & $27(28,4)$ & $56(32,9)$ \\
\hline & Más de 3 minutos & $46(61,3)$ & $68(71,6)$ & $114(67,1)$ \\
\hline \multirow{4}{*}{$\begin{array}{l}\text { 5. En qué momento del día } \\
\text { cepillas tus dientes }\end{array}$} & Mañana y noche & $40(53,3)$ & $52(54,7)$ & $92(54,1)$ \\
\hline & Sólo mañana & $10(13,3)$ & $8(8,4)$ & $18(10,6)$ \\
\hline & Sólo noche & $4(5,3)$ & $1(1,1)$ & $5(2,9)$ \\
\hline & Después de cada comida & $21(28,0)$ & $34(35,8)$ & $55(32,4)$ \\
\hline \multirow{5}{*}{$\begin{array}{l}\text { 6. Cada cuánto tiempo cambias } \\
\text { el cepillo de dientes }\end{array}$} & $1-3$ meses & $49(65,3)$ & $60(63,2)$ & $109(64,1)$ \\
\hline & 4-6 meses & $8(10,7)$ & $17(17,9)$ & $25(14,7)$ \\
\hline & 7-12 meses & $6(8,0)$ & $2(2,1)$ & $8(4,7)$ \\
\hline & Después de un año & $1(1,3)$ & $3(3,2)$ & $4(2,4)$ \\
\hline & No lo sé & $11(14,7)$ & $12(12,6)$ & $23(13,5)$ \\
\hline \multirow[t]{3}{*}{ 7. Técnica de cepillado } & Movimientos horizontales (de lado a lado) & $31(41,3)$ & $19(20,0)$ & $50(29,4)$ \\
\hline & Movimientos de arriba abajo & $39(52,0)$ & $63(66,3)$ & $102(60,0)$ \\
\hline & No sigo ninguna & $5(6,7)$ & $13(13,7)$ & $18(10,6)$ \\
\hline
\end{tabular}


un período de 1 a 3 meses. En la Tabla 5 se muestran las prácticas en salud oral que tienen los adolescentes distribuidos por edad.

\section{DISCUSIÓN}

La información en cuanto al conocimiento, las actitudes y prácticas de los adolescentes sobre la salud oral, son datos importantes para la planificación de promociones efectivas, considerando las necesidades particulares del grupo a intervenir. Estas promociones deberían enfocarse en entregar herramientas que permitan a los jóvenes identificar las enfermedades orales más comunes, saber cómo evitarlas por medio del autocuidado, y que lleven a la práctica diaria lo aprendido, para que de esta manera mantengan un buen estado de salud oral.

Los resultados de este estudio mostraron que la población adolescente de 12 y 15 años de Fresia tiene un nivel de conocimiento similar a jóvenes de otras regiones del mundo. Un alto porcentaje tiene conocimientos de las causas de la caries dental, identificando como factor causal las bacterias y dulces, similar a lo reportado en India y Suecia..$^{8,9}$ Sin embargo, el conocimiento en cuanto a los métodos de prevención, la mayoría solo identifica como útil en la prevención de la caries al cepillo dental, al igual que Gupta et al. y Al-Omiri et al., 810 donde los encuestados tenían conocimiento sobre el rol del cepillado dental, pero identificando además otros elementos de higiene como coadyuvantes en la prevención de la caries. Por otro lado, adolescentes suecos con alto riesgo de caries consideraron un mal cepillado dental como un factor de riesgo de caries. $^{9}$

En cuanto al rol preventivo del hilo dental y del flúor, los jóvenes encuestados mostraron un escaso conocimiento, repitiéndose esta situación en distintos estudios. ${ }^{8,9,11}$ Con respecto a la enfermedad de las encías (gingivitis), solo un $69,4 \%$ consideró la falta de higiene como factor causal, a diferencia de lo mostrado en relación a la caries, $(81 \%)$ esto se podría relacionar con el poco conocimiento del rol preventivo del cepillado dental en la gingivitis, similar a lo observado por Gupta et al., ${ }^{8}$ donde solo el $50-60 \%$ de los escolares, tenía conocimiento de los signos y causas relacionadas con esta enfermedad. Por otra parte, en un estudio realizado en Suecia, los encuestados no relacionaron en lo absoluto una mala higiene oral con malas condiciones periodontales. ${ }^{9}$ Por su parte, Al-Omiri et al. ${ }^{10}$ encontró que un $70 \%$ de los adolescentes tenía conocimiento del sangrado de las encías como reflejo de la gingivitis, similar a lo reportado en este estudio, donde el 75,9\%, identificó el sangrado de las encías como signo de enfermedad.

Se ha visto que posponer la visita al dentista y resignarse a tener caries, se vuelve más predominante a medida que aumenta el nivel de escolaridad. ${ }^{12}$ De los encuestados en Fresia, gran parte muestra conocimiento sobre la importancia de visitar al dentista, aunque no presenten dolor, a diferencia de lo obtenido por Gupta et al., donde los participantes declararon visitar al dentista solo en caso de dolor en los dientes o encías, ${ }^{8}$ resultados prometedores, ya que visitar al dentista solo por control, puede asegurar una mejor mantención de la salud oral.

En cuanto a las actitudes relacionadas con la salud oral, la mayoría de los adolescentes de 12 años declara tener un cuidado regular de sus dientes, no así los de 15 años, los cuales creen tener un buen cuidado de sus dientes, lo que contrasta con lo reportado por Ericsson et al., ${ }^{6}$ donde el $90 \%$ de los adolescentes consideró su salud oral como buena o muy buena. Sin embargo, frente a las enfermedades de las encías, la mayoría de los jóvenes de Fresia calificó su conocimiento como bueno y regular, teniendo una mejor autovaloración que los encuestados por Ericsson et al. ${ }^{6}$ Un $68 \%$ consideró muy importante tener condiciones bucales saludables, siendo los propios esfuerzos el factor más importante para el futuro de su salud oral, similar a lo reportado por jóvenes suecos. ${ }^{6}$ De acuerdo a esto, al reconocer su responsabilidad en la mantención de su salud oral, es posible pensar en el impacto positivo que podrían tener las promociones enfocadas en el autocuidado.

De las prácticas relacionadas con salud oral, la totalidad de los adolescentes encuestados declaró utilizar pasta dental para su 
cepillado, sin embargo, más de la mitad no sabe si su pasta dental es o no fluorada, por lo que podríamos pensar que no conocen el rol preventivo de la pasta dental. El cepillado dental es realizado principalmente durante la mañana y noche, con una duración mayor a tres minutos, similar a lo reportado en otros estudios, ${ }^{6,10,11}$ lo cual sugeriría que los jóvenes no han adquirido el hábito de cepillarse después de cada comida, lo que podría deberse a que la mayoría almuerza en los establecimientos educacionales y no lleva su cepillo dental. La principal técnica de cepillado utilizada por los jóvenes de 12 años, es con movimientos horizontales y en los jóvenes de 15 años con movimientos de arriba a abajo; llama la atención la realización incorrecta de la técnica de cepillado en los jóvenes de 12 años, a pesar de haber recibido educación en prevención, lo cual indicaría la poca adherencia a las prácticas enseñadas.

A pesar de existir conciencia en ciertos cuidados de salud oral por estos jóvenes, se hace necesario realizar métodos educativos donde los contenidos sean dirigidos de una manera amigable, con participación y demostración individual, lo cual debiera considerarse en futuras promociones dirigidas a este grupo etario, para lograr un impacto positivo, sin dejar de considerar la realización de un seguimiento a los jóvenes intervenidos, ya que de acuerdo a lo reportado por Ericsson et al., un mayor conocimiento no da lugar de manera automática a un cambio de comportamiento más beneficioso en salud bucal.

Como limitación del estudio, consideramos que la información obtenida de las respuestas, al ser recopilada por medio de una encuesta, podría estar sesgada, no mostrando la realidad de la población encuestada. Se recomienda como futura línea investigativa realizar una encuesta similar a la presentada, pero con un examen clínico previo, de manera de poder comparar el nivel de conocimiento, actitudes y prácticas, con el real estado de salud oral de los encuestados.

De acuerdo a estos resultados, podemos concluir que los adolescentes de 12 y 15 años de la localidad de Fresia, tienen un nivel de conocimiento de salud oral aceptable, sin embargo, se debe reforzar la educación de las enfermedades que afectan a las encías. En relación a las actitudes y prácticas, los resultados son favorables.

\section{AGRADECIMIENTOS}

Los autores agradecen a la Ilustre Municipalidad de Fresia y a los directores de los diferentes establecimientos educacionales de la localidad.

\section{REFERENCIAS}

1. Organización Mundial de la Salud. Encuestas de Salud Bucodental. Métodos Básicos. ${ }^{a}$ ed. Ginebra: OMS; 1997.

2. Cueto U, Soto C, Muñoz D. Conocimiento, Actitudes y Práctica sobre Salud Oral en Niños de 12 años. Rev Chil Salud Pública. 2010; 14(2-3): 361-3.

3. Saliba C, Dossi de Guimarães A, Ísper A, Saliba S, Barreto G. (2013) Comparison of methods in oral health education from the perspective of adolescents. Oral Health Prev Dent. 2013; 11: 39-47.

4. Misrachi C, Arellano M. Conductas y factores determinantes en salud oral de los adolescentes. Rev Chil Pediatr. 1995;66(6): 317-22.

5. Oliveira E, Narendran S, Williamson D. Oral health knowledge, attitudes and preventive practices of third grade school children. Pediatr Dent. 2000; 22: 395-400.

6. Ericsson J, Östberg A, Wennstrom J, Abrahamsson K. Oral health-related perceptions, attitudes and behavior in relation to oral hygiene conditions in an adolescent population. Eur J Oral Sci. 2012; 120: 335-41.

7. Brukiene V, Aleksejuniene J. An overview of oral health promotion in adolescents. Int J Pediatr Dent. 2009; 19: 163-71.

8. Gupta T, Sequeira P, Acharya S. Oral health knowledge, attitude and practices of a 15-year-old adolescent population in Southern India and their social determinants. Oral Health Prev Dent. 2012;10: 345-54.

9. Hattne K, Folke S, Twetman S. Attitudes to oral health among adolescents with high 
caries risk. Acta Odontol Scand. 2007 Aug; 65(4): 206-13.

10. Al-Omiri M, Al-Wahadni A, Saeed K. Oral health attitudes, knowledge and behavior among school children in North Jordan. $J$ Dent Educ. 2006; 70(2): 179-87.

11. Suprabha B, Rao A, Shenoy R, Khanal S. Utility of knowledge, attitude, and prac- tice survey, and prevalence of dental caries among 11-to 13-year-old children in an urban community in India. Glob Health Action. 2013; 6: 20750.

12. Kawamura M, Takase N, Okada M. Teenagers' oral health attitudes and behavior in Japan: comparison by sex and age group. $J$ Oral Sci. 2008; 50(2): 167-74.

\section{ANEXO}

Tabla 1. Encuesta conocimientos, actitudes y prácticas en salud oral en adolescentes de 12 y 15 años (adaptado de Gupta et al., 2012)

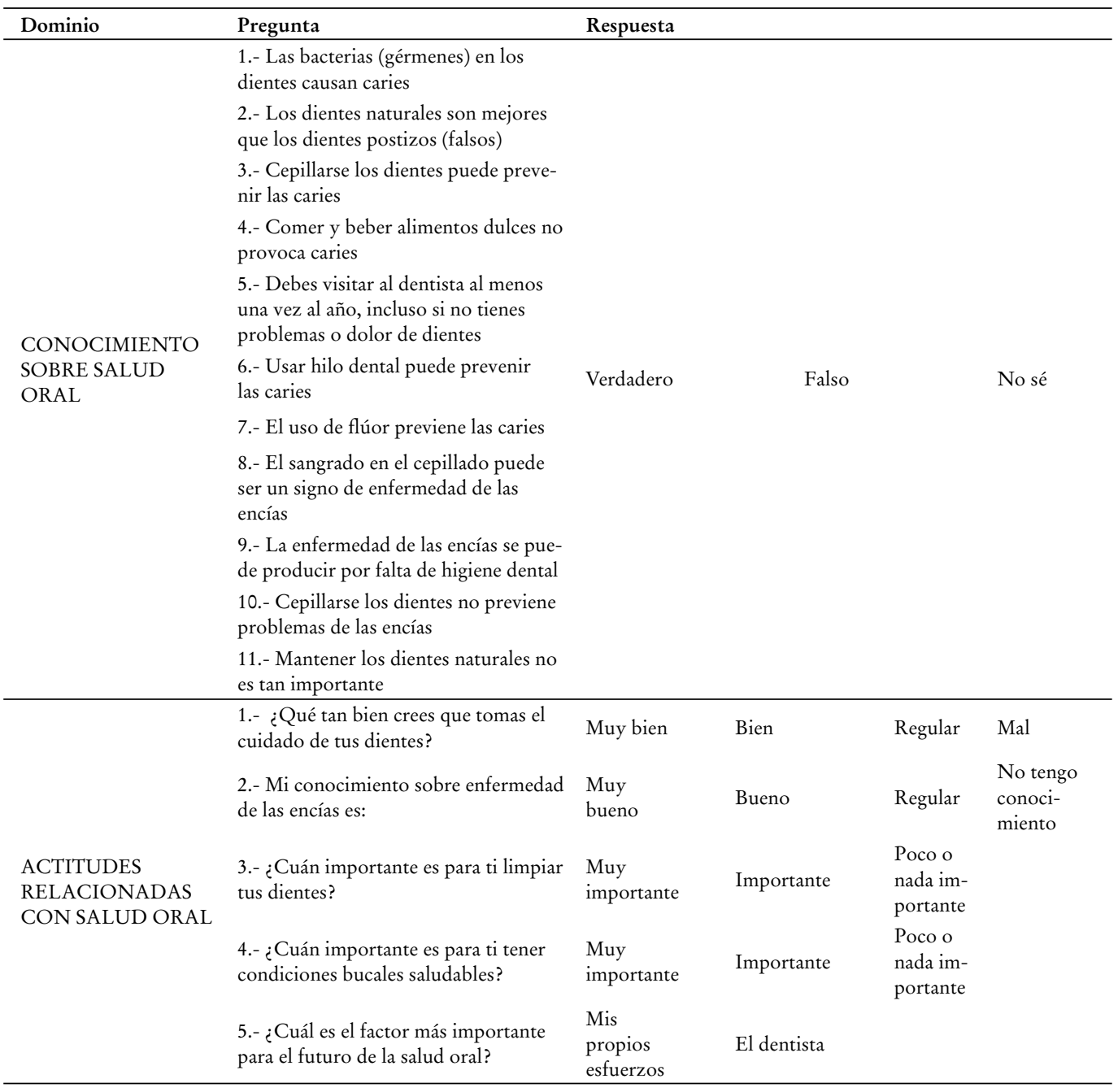




\begin{tabular}{|c|c|c|c|c|c|c|}
\hline \multirow{7}{*}{$\begin{array}{l}\text { PRÁCTICAS } \\
\text { RELACIONADAS } \\
\text { CON SALUD ORAL }\end{array}$} & 1.- Uso del cepillo de dientes & Sí & No & & & \\
\hline & 2.- Elementos utilizados para cepillarse & Agua & $\begin{array}{l}\text { Pasta de } \\
\text { dientes }+ \\
\text { agua }\end{array}$ & Nada & & \\
\hline & 3.- Tipo de pasta de diente & Fluorada & $\begin{array}{l}\text { No Fluo- } \\
\text { rada }\end{array}$ & No lo sé & & \\
\hline & $\begin{array}{l}\text { 4.- Tiempo de duración del cepillado } \\
\text { de dientes }\end{array}$ & $\begin{array}{l}\text { Menos de } \\
3 \text { minutos }\end{array}$ & $\begin{array}{l}\text { Más de } 3 \\
\text { minutos }\end{array}$ & & & \\
\hline & $\begin{array}{l}\text { 5.- En qué momento del día cepillas } \\
\text { tus dientes }\end{array}$ & $\begin{array}{l}\text { Mañana y } \\
\text { noche }\end{array}$ & $\begin{array}{l}\text { Sólo } \\
\text { mañana }\end{array}$ & $\begin{array}{l}\text { Solo } \\
\text { noche }\end{array}$ & $\begin{array}{l}\text { Después } \\
\text { de cada } \\
\text { comida }\end{array}$ & \\
\hline & $\begin{array}{l}\text { 6.- Cada cuanto tiempo cambias el } \\
\text { cepillo de dientes }\end{array}$ & 1 a 3 meses & 4-6 meses & $\begin{array}{l}7-12 \\
\text { meses }\end{array}$ & $\begin{array}{l}\text { Después } \\
\text { de un año }\end{array}$ & No lo sé \\
\hline & 7.- Técnica de cepillado & $\begin{array}{l}\text { Movi- } \\
\text { mientos } \\
\text { horizonta- } \\
\text { les (lado a } \\
\text { lado) }\end{array}$ & $\begin{array}{l}\text { Movi- } \\
\text { mientos } \\
\text { de arriba } \\
\text { abajo }\end{array}$ & $\begin{array}{l}\text { No sigo } \\
\text { ninguna }\end{array}$ & & \\
\hline \multirow{3}{*}{$\begin{array}{l}\text { DATOS } \\
\text { PERSONALES }\end{array}$} & 1.- Sexo & Masculino & Femenino & & & \\
\hline & 2.- Edad & En años & & & & \\
\hline & 3.- Residencia & Urbano & Rural & & & \\
\hline
\end{tabular}

